\title{
Glucose-responsive insulin and glucagon delivery (dual-hormone artificial pancreas) in adults with type 1 diabetes: a randomized crossover controlled trial
}

\author{
Ahmad Haidar Eng MScA, Laurent Legault MD, Maryse Dallaire RN, Ammar Alkhateeb MD, \\ Adèle Coriati MSc, Virginie Messier MSc, Peiyao Cheng MPH, Maude Millette MD, Benoit Boulet Eng PhD, \\ Rémi Rabasa-Lhoret MD PhD
}

See related commentary by Nathan and Russell on page 285 and at www.cmaj.ca/lookup/doi/10.1503/cmaj.130011

\begin{abstract}
- Abstract
Background: Most patients with type 1 diabetes do not achieve their glycemic targets. We aimed to assess the efficacy of glucoseresponsive insulin and glucagon closed-loop delivery for controlling glucose levels in adults with type 1 diabetes.

Methods: We conducted a randomized crossover trial involving 15 adults with type 1 diabetes, comparing standard insulin-pump therapy with dual-hormone, closed-loop delivery. Patients were admitted twice to a clinical research facility and received, in random order, both treatments. Each 15-hour visit (from 1600 to 0700 ) included an evening exercise session, followed by a medium-sized meal, a bedtime snack and an overnight stay. During visits that involved closed-loop delivery, basal insulin and glucagon miniboluses were delivered according to recommendations based on glucose sensor readings and a predictive dosing algorithm at 10 -minute intervals. During visits involving standard insulin-pump therapy (control visits), patients used conventional treatment.
\end{abstract}

Results: Dual-hormone closed-loop delivery increased the percentage of time for which patients' plasma glucose levels were in the target range (median $70.7 \%$ [interquartile range (IQR) $46.1 \%-88.4 \%$ ] for closed-loop delivery v. $57.3 \%$ [IQR 25.2\%-71.8\%]for control, $p=0.003$ ) and decreased the percentage of time for which plasma glucose levels were in the low range (bottom of target range $[<4.0 \mathrm{mmol} / \mathrm{L}], 0.0 \%$ [IQR $0.0 \%-3.0 \%$ ] for closed-loop delivery v. $10.2 \%$ [IQR $0.0 \%-13.0 \%$ ] for control, $p=0.01$ hypoglycemia threshold [ $<3.3 \mathrm{mmol} / \mathrm{L}], 0.0 \%$ [IQR $0.0 \%-0.0 \%$ ] for closed-loop delivery v. $2.8 \%$ [IQR $0.0 \%-5.9 \%$ ] for control, $p=0.006$ ). Eight participants $(53 \%)$ had at least 1 hypoglycemic event (plasma glucose $<3.0 \mathrm{mmol} / \mathrm{L}$ ) during standard treatment, compared with just 1 participant (7\%) during closed-loop treatment $(p=0.02)$.

Interpretation: Dual-hormone, closed-loop delivery guided by advanced algorithms improved short-term glucose control and reduced the risk of hypoglycemia in a group of 15 adults with type 1 diabetes. Trial registration: ClinicalTrials.gov, no. NCT01297946.
$\mathrm{T}$ he Diabetes Control and Complications Trial has shown that intensive insulin therapy in type 1 diabetes with the aim of good glycemic control substantially reduces microvascular and macrovascular complications. ${ }^{1,2}$ However, despite advances in insulin analogs, insulin pumps and continuous glucosemonitoring systems, glucose control remains problematic, and most patients with type 1 diabetes do not achieve their glycemic targets. ${ }^{3}$

Hypoglycemia remains the major barrier to the intensification of insulin therapy. ${ }^{4}$ Intensive insulin therapy and lower levels of glycated hemoglobin are unfortunately associated with an increased risk of hypoglycemia. ${ }^{5}$ The frequency of patient-reported nonsevere hypoglycemia (blood glucose $\leq 3.5 \mathrm{mmol} / \mathrm{L}$, with or without symptoms) is about 2.7 episodes/patient per week, ${ }^{6}$ with episodes commonly occuring during the night. In a recent continuous glucosemonitoring trial conducted by the Juvenile Diabetes Research Foundation, ${ }^{7}$ hypoglycemia (glucose sensor reading $<3.3 \mathrm{mmol} / \mathrm{L}$ ) occurred during $8.5 \%$ of the nights included in the study
Competing interests: Ahmad Haidar and Rémi Rabasa-Lhoret have a patent pending for a closed-loop system dosing algorithm. Laurent Legault has received grant funding from Diabète Québec. Laurent

Legault, Benoit Boulet and Rémi Rabasa-Lhoret have received grant funding from the Canadian Diabetes Association. Rémi Rabasa-

Lhoret is a consultant for AstraZeneca, Boehringer, Eli Lilly, Merck, NovoNordisk and Sanofi-Aventis; he has received grants from AstraZeneca, Eli Lilly,

Merck, Novo-Nordisk and Sanofi-Aventis; he has received speaker fees from AstraZeneca, Eli Lilly, Merck and Novo-Nordisk.

This article has been peer reviewed.

Correspondance to:

Ahmad Haidar, ahmad.haidar@mail.mcgill. ca

CMAJ 2013. DOI:10.1503 /cmaj.121265 
period, with $47 \%$ of those nights involving at least 1 hour of hypoglycemia, $23 \%$ involving at least 2 hours, and $11 \%$ involving at least 3 hours.

Advances in insulin infusion pumps and continuous glucose-monitoring systems could improve glycemic control $;{ }^{8}$ however, we still lack the ability to combine these devices in an automated manner. Closed-loop insulin delivery systems (i.e., the artificial pancreas) combine the 2 devices using a mathematical algorithm. ${ }^{9}$ These systems might improve glycemic control and reduce the risk of hypoglycemia compared with conventional insulin-pump therapy (i.e., continuous subcutaneous insulin infusion)..$^{10,11}$ However, a clinically significant number of hypoglycemic events (blood glucose < $3.0 \mathrm{mmol} / \mathrm{L}$ ) were still reported during tests of closed-loop delivery systems. ${ }^{10,11}$

Dual-hormone closed-loop delivery systems have also been proposed to regulate glucose levels. These systems combine insulin delivery with subcutaneous glucagon delivery to further reduce the risk of hypoglycemia. ${ }^{12-14}$ However, their potential benefits to improve glycemic control are currently unknown. We sought to determine whether dual-hormone closed-loop delivery, compared with conventional insulin pump therapy, can improve glycemic control and reduce the risk of hypoglycemia in adults with type 1 diabetes.

\section{Methods}

\section{Study design}

We used an open-label, randomized, crossover design to compare dual-hormone closed-loop delivery with continuous subcutaneous insulin infusion (the control) in adults with type 1 diabetes. Each study visit included an evening exercise session, followed by a meal, a bedtime snack and an overnight stay. The 2 interventions were separated by 7 (interquartile range [IQR] 3-14) days.

\section{Participants}

From February 2011 to January 2012, we enrolled participants from the diabetes clinic at the Institut de Recherches Cliniques de Montréal (Montréal, Quebec). Participants were required to be more than 18 years of age and to have been using an insulin pump for at least 3 months. Patients whose diabetes was poorly controlled (glycated hemoglobin $>10 \%$ ) were excluded. Other exclusion criteria were applied as detailed in Appendix 1 (available at www.cmaj.ca/lookup /suppl/doi:10.1503/cmaj.121265/-/DC1). All participants provided their written informed consent. The study was approved by the research ethics committee at the Institut de Recherches Cliniques de Montréal.

\section{Sample size and randomization}

We anticipated that dual-hormone closed-loop delivery would increase the percentage of time for which plasma glucose concentrations are in the target range by $22 \%$ (standard deviation $[\mathrm{SD}]=22 \%$ ). We calculated that 10 participants would provide $80 \%$ power at the $5 \%$ level of significance to detect such a difference between the 2 interventions. The study included, by its design, an interim assessment to evaluate the appropriateness of the sample size after 6 participants had completed both arms (i.e., a total of 12 completed visits). Subsequently, we adjusted the number needed to enrol to 16 participants, one of whom did not complete the study (Figure 1).

We used blocked randomization with an equal allocation ratio to generate allocation sequences. Patients were not blinded to the allocation. Blinding was practically challenging, because patients had to control their glucose levels during control visits, but not during dual-hormone closed-loop delivery.

\section{Study protocol}

Participants arrived at the research facility at about 1500 and received treatment from 1600 until 0700 the next day.

The exercise session consisted of a 30-minute workout on a stationary bicycle at $60 \% V \mathrm{O}_{\text {max }}$ (each patient's $V \mathrm{O}_{2 \max }$ had been determined before randomization). At 1730, participants' capillary glucose levels were checked using a glucose meter. The exercise session began at 1750 if the glucose level was above $6.0 \mathrm{mmol} / \mathrm{L}$. If the glucose level was below $6.0 \mathrm{mmol} / \mathrm{L}, 15 \mathrm{~g}$ carbohydrate was given orally, and the exercise session began once the glucose level was above $6.0 \mathrm{mmol} / \mathrm{L}$.

Each participant received a standardized meal (60 g carbohydrate for females, $80 \mathrm{~g}$ carbohydrate for males) at 1920 and a bedtime snack ( $15 \mathrm{~g}$ carbohydrate) at 2200 (for a description of the meal and snack, see Appendix 2, available at www.cmaj.ca/lookup/suppl/doi: 10.1503/cmaj.121265/-/DC1). Participants were blinded to their plasma and sensor glucose data during both visits and to the hormonal infusions during closed-loop visits.

We drew venous blood samples every 10 30 minutes to determine plasma glucose and insulin levels, and every 10-60 minutes to determine plasma glucagon levels. Plasma glucose levels were measured using a YSI2300 STAT Plus Analyzer (Yellow Springs, Ohio). Plasma insulin and glucagon were measured using an immunoassay (Millipore, Billerica, Massachusetts). 
During visits involving dual-hormone closedloop delivery, glucose levels were regulated using variable subcutaneous insulin delivery combined with subcutaneous miniboluses of glucagon. Insulin aspart and recombinant glucagon were delivered by 2 infusion pumps (MiniMed Paradigm Veo, Medtronic, Northridge, California), according to recommendations, at 10-minute intervals and as determined by our dosing algorithm (Appendix 2). At the time of the evening meal, we entered the meal's carbohydrate content into the algorithm to calculate the prandial bolus. Insulin and glucagon delivery was otherwise based only on readings from a continuous glucose sensor measuring interstitial glucose (Sofsensor, Medtronic).

Every 10 minutes, we manually entered the real-time readings from the continuous glucose sensor into a computer that calculated insulin and glucagon delivery. We then manually gave the insulin and glucagon through the infusion pumps. We used a single sensor that we calibrated using finger-stick capillary glucose measurements. We did not recalibrate or replace the sensor in the event of suboptimal accuracy, and we always adhered to the dosing algorithm.

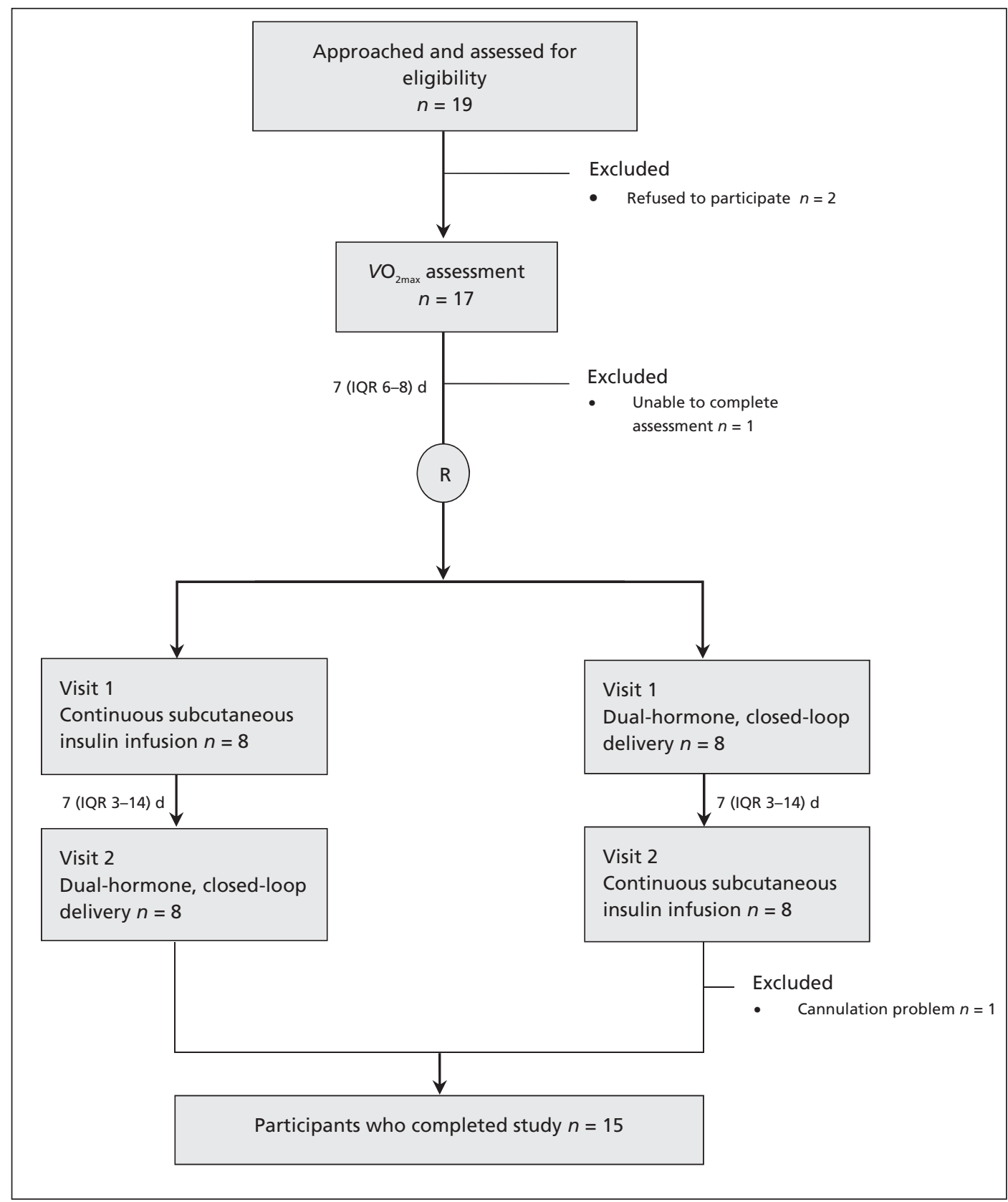

Figure 1: Flow of participants through the crossover study, showing the crossover in therapies between visits 1 and 2 . The crossed-over streams are shown in parallel on each side of the flow chart. IQR = interquartile range, $\mathrm{VO}_{2 \max }=$ maximum oxygen uptake. 
A

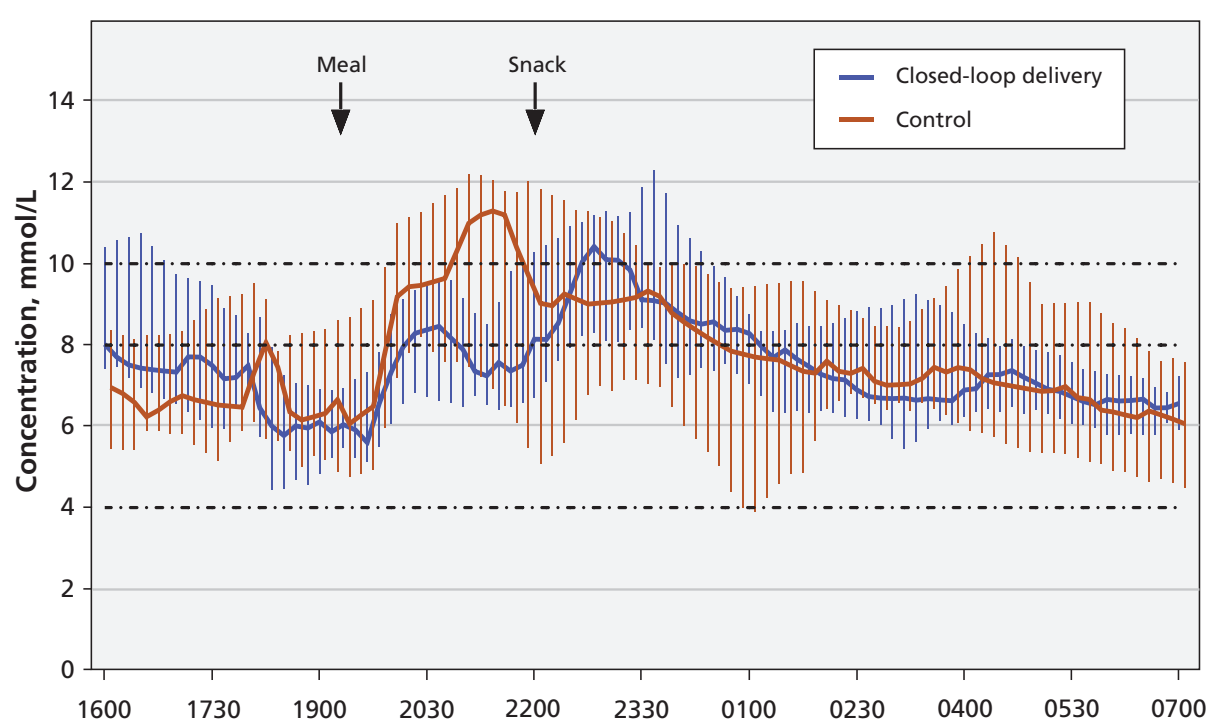

B

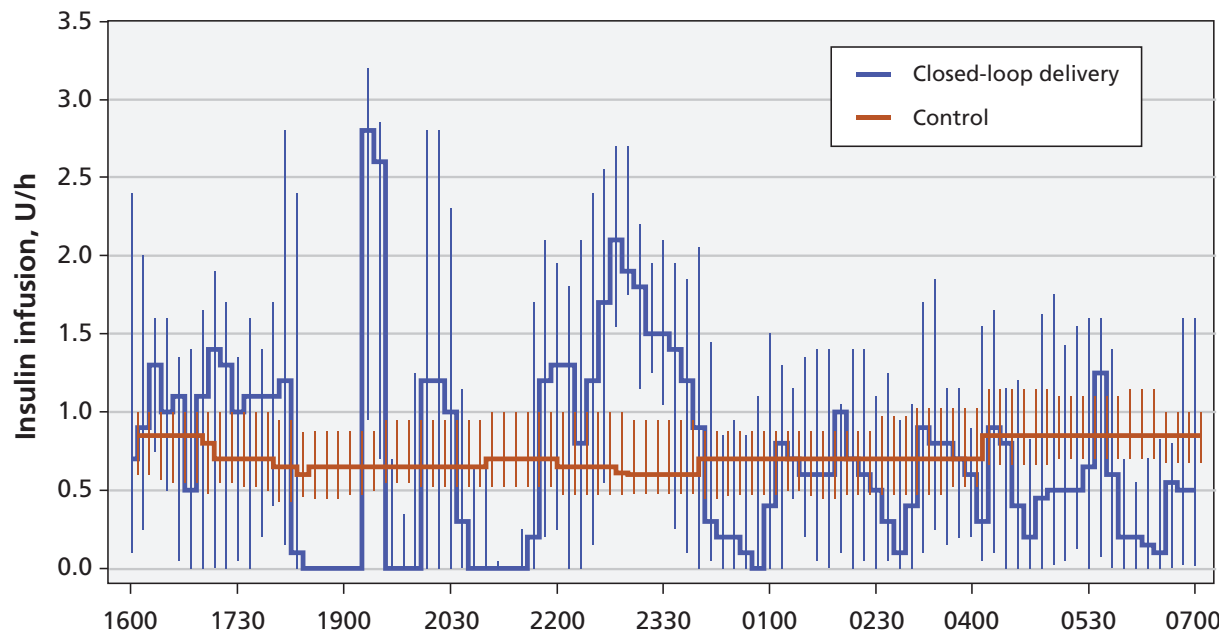

C

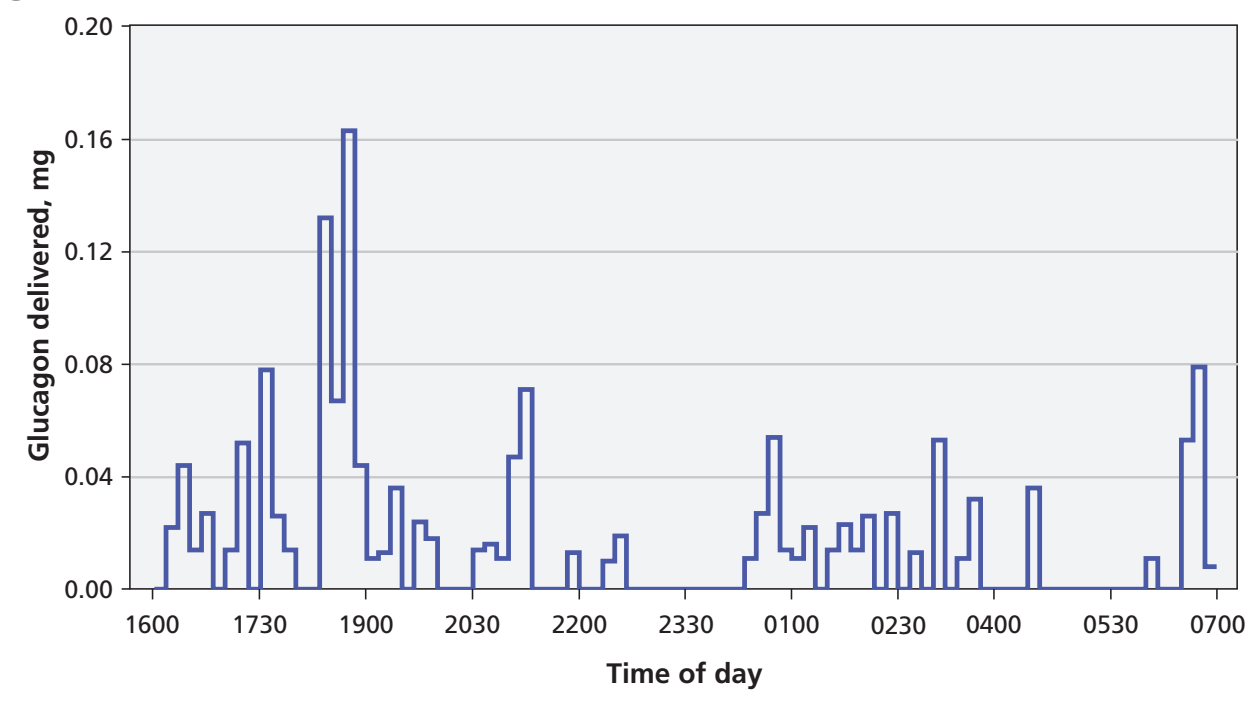

Figure 2: Profiles (medians and interquartile ranges) of (A) plasma glucose concentration and (B) basal insulin infusion with dual-hormone closed-loop delivery and continuous subcutaneous insulin infusion. (C) The histogram of glucagon delivery during closed-loop delivery. 
During control visits, participants received continuous subcutaneous insulin infusion. Participants had access to their finger-stick glucose measurements, knew the carbohydrate content of their meal and snack, and consequently adjusted their insulin delivery (including temporary basal and correction boluses) as per their standard practice.

\section{Outcomes measures}

Our primary outcome was the percentage of time for which plasma glucose concentrations were in the target range during each 15-hour visit (4.00$10.00 \mathrm{mmol} / \mathrm{L}$ between the hours of 1600 and 2300, and 4.00-8.00 $\mathrm{mmol} / \mathrm{L}$ between the hours of 2300 and 0700 ).

Our secondary outcomes were the percentage of time spent below the target range, the percentage of time spent above the target range, the total amount of insulin delivered, the standard deviation of plasma glucose concentrations and the number of participants with hypoglycemic events (blood glucose $<3.0 \mathrm{mmol} / \mathrm{L}$ ).

\section{Statistical analysis}

For each continuous outcome, we used a repeated-measures regression model based on the ranked normal transformation (with the exception of mean glucose, which was not transformed) to compare the 2 treatments, adjusting for the starting glucose level and the period effect. We used the McNemar test to compare rates of hypoglycemia.

\section{Results}

Fifteen participants completed the study and were included in our analysis (Figure 1). The

Table 1: Comparison of outcomes among 15 adults with type 1 diabetes receiving both interventions

\begin{tabular}{|c|c|c|c|}
\hline \multirow[b]{2}{*}{ Outcome } & \multicolumn{2}{|c|}{ Intervention, median (IQR)* } & \multirow[b]{2}{*}{$p$ valuet } \\
\hline & $\begin{array}{l}\text { Dual-hormone } \\
\text { closed-loop delivery }\end{array}$ & $\begin{array}{l}\text { Continuous } \\
\text { subcutaenous } \\
\text { insulin infusion }\end{array}$ & \\
\hline \multicolumn{4}{|l|}{$\begin{array}{l}\text { Overall (duration of visit, from } 1600 \text { to } \\
\text { 0700) }\end{array}$} \\
\hline Plasma glucose level at start of visit, $\mathrm{mmol} / \mathrm{L}$ & $7.7(7.4-10.6)$ & $7.0(5.2-8.7)$ & 0.28 \\
\hline \multicolumn{4}{|l|}{ Time spent at specific glucose level, $\%$} \\
\hline Target range $\ddagger$ & $70.7(46.1-88.4)$ & $57.3(25.2-71.8)$ & 0.003 \\
\hline$<4.0 \mathrm{mmol} / \mathrm{L}$ & $0.0(0.0-3.0)$ & $10.2(0.0-13.0)$ & 0.01 \\
\hline$<3.3 \mathrm{mmol} / \mathrm{L}$ & $0.0(0.0-0.0)$ & $2.8(0.0-5.9)$ & 0.006 \\
\hline Above target range & $29.3(9.4-49.2)$ & $25.6(18.0-63.4)$ & 0.17 \\
\hline Plasma glucose level, $\mathrm{mmol} / \mathrm{L}$, mean $\pm \mathrm{SD}$ & $7.8 \pm 1.1$ & $7.9 \pm 1.9$ & 0.74 \\
\hline Plasma glucose level, mmol/L, SD & $2.0(1.3-2.8)$ & $2.1(1.8-2.9)$ & 0.06 \\
\hline Insulin delivery, U & $19(15-23)$ & $19(15-25)$ & 0.19 \\
\hline Insulin concentration, mU/L & $25(23-42)$ & $30(18-43)$ & 0.87 \\
\hline Glucagon concentration, $\mathrm{pg} / \mathrm{mL}$ & $66(61-72)$ & $56(43-65)$ & 0.01 \\
\hline \multicolumn{4}{|l|}{ Overnight (from 2300 to 0700 ) } \\
\hline \multicolumn{4}{|l|}{ Time spent at specific glucose level, \% } \\
\hline Target range & $72.0(32.5-84.2)$ & $45.8(29.2-64.6)$ & 0.07 \\
\hline$<4.0 \mathrm{mmol} / \mathrm{L}$ & $0.0(0.0-0.0)$ & $0.0(0.0-22.5)$ & 0.02 \\
\hline$<3.3 \mathrm{mmol} / \mathrm{L}$ & $0.0(0.0-0.0)$ & $0.0(0.0-7.7)$ & 0.01 \\
\hline Above target range & $28.0(15.8-64.8)$ & $49.8(12.5-70.8)$ & 0.99 \\
\hline Plasma glucose level, $\mathrm{mmol} / \mathrm{L}$, mean $\pm \mathrm{SD}$ & $7.8 \pm 1.3$ & $7.6 \pm 2.3$ & 0.63 \\
\hline Plasma glucose level, mmol/L, SD & $1.5(1.1-2.6)$ & $1.6(1.1-2.1)$ & 0.67 \\
\hline Insulin delivery, U & $14(10-16)$ & $14(9-17)$ & 0.77 \\
\hline Insulin concentration, mU/L & $24(19-31)$ & $25(15-33)$ & 0.68 \\
\hline Glucagon concentration, $\mathrm{pg} / \mathrm{mL}$ & $57(45-70)$ & $50(37-61)$ & 0.02 \\
\hline $\begin{array}{l}\text { Note: } I Q R=\text { interquartile range, } S D=\text { standard deviatio } \\
\text { *Unless stated otherwise. } \\
\text { †Repeated measures analysis. } \\
\text { †Primary study outcome. }\end{array}$ & & & \\
\hline
\end{tabular}


characteristics of the participants are listed in Appendix 3 (available at www.cmaj.ca/lookup /suppl/doi:10.1503/cmaj.121265/-/DC1).

Dual-hormone closed-loop delivery generally reduced interpatient variability in plasma glucose concentrations (Figure 2). The median and IQRs of participants' glucose levels during control visits suggest that, during the night, $25 \%$ of the participants had glucose levels below $4.0 \mathrm{mmol} / \mathrm{L}$ at about 0100 , and $25 \%$ of the participants had glucose levels above $10.0 \mathrm{mmol} / \mathrm{L}$ at about 0530 (Figure 2).

During visits involving closed-loop delivery, participants' plasma glucose levels were in the target range a median of $70.7 \%$ of the time (IQR $46.4 \%-88.4 \%$ ), compared with $57.3 \%$ of the time (IQR $25.2 \%-71.8 \%$ ) during control visits $(p=0.003$; Table 1). Compared with conventional treatment, closed-loop delivery significantly decreased the percentage of time spent in hypoglycemia $(0.0 \%$ v. $10.2 \%$ for plasma glucose levels $<4.0 \mathrm{mmol} / \mathrm{L}$, and $0.0 \%$ v. $2.8 \%$ for plasma glucose levels $<3.3 \mathrm{mmol} / \mathrm{L}$; Table 1 ).

The percentage of time spent in the target range for plasma glucose levels during the night was higher for closed-loop delivery than for conventional treatment $(72.0 \%$ v. $45.8 \%$, repeated measures analysis $p=0.07$, Table 1 ). Closedloop delivery also reduced nocturnal hypoglycemia. When samples collected during the

Table 2: Rates of hypoglycemia and nocturnal plasma glucose concentrations among 15 patients with type 1 diabetes during each intervention

\begin{tabular}{|c|c|c|c|}
\hline \multirow[b]{2}{*}{ Outcome } & \multicolumn{2}{|c|}{ Intervention, no. (\%) } & \multirow[b]{2}{*}{$p$ value* } \\
\hline & $\begin{array}{l}\text { Dual-hormone } \\
\text { closed-loop } \\
\text { delivery } \\
n=15\end{array}$ & $\begin{array}{c}\text { Continuous } \\
\text { subcutaneous } \\
\text { insulin infusion } \\
n=15\end{array}$ & \\
\hline $\begin{array}{l}\text { Patients with at least } 1 \\
\text { hypoglycemic event } t\end{array}$ & $1(7)$ & $8(53)$ & 0.02 \\
\hline $\begin{array}{l}\text { Patients with at least } 1 \\
\text { nocturnal hypoglycemic } \\
\text { event†‡ }\end{array}$ & $0(0)$ & $5(33)$ & 0.07 \\
\hline $\begin{array}{l}\text { Patients with at least } 1 \\
\text { exercise-induced } \\
\text { hypoglycemic event }+\S\end{array}$ & $1(7)$ & $2(13)$ & 1.0 \\
\hline $\begin{array}{l}\text { Nocturnal‡ plasma glucose } \\
\text { measurements }\end{array}$ & $n=363$ & $n=367$ & \\
\hline$<4.0 \mathrm{mmol} / \mathrm{L}$ & $2(0.5)$ & $45(12.3)$ & - \\
\hline$<3.3 \mathrm{mmol} / \mathrm{L}$ & $0(0.0)$ & $13(3.5)$ & - \\
\hline$<3.0 \mathrm{mmol} / \mathrm{L}$ & $0(0.0)$ & 9 (2.5) & - \\
\hline \multicolumn{4}{|c|}{$\begin{array}{l}\text { *McNemar test. } \\
\text { †Defined as at least } 1 \text { measurement of plasma glucose concentration }<3.0 \mathrm{mmol} / \mathrm{L} \text {, which } \\
\text { was treated by oral carbohydrate. } \\
\text { †Between the hours of } 2300 \text { and } 0700 \text {. } \\
\text { \$From start of exercise to } 1920 . \\
\text { १Measurements were taken at } 20 \text {-min intervals. }\end{array}$} \\
\hline
\end{tabular}

night were combined (Table 2), 45 (12.3\%) of the measurements of plasma glucose concentration were below $4.0 \mathrm{mmol} / \mathrm{L}$ during control visits, compared with only 2 measurements $(0.5 \%)$ during closed-loop delivery, a more than 20 -fold reduction.

The total amounts of insulin delivered did not differ between the 2 interventions (Table 1). Prandial boluses, as determined using our algorithm, were lower by 0.9 (IQR 0.6-2.2) units during visits involving closed-loop delivery, compared with patient-determined boluses during control visits (repeated measures analysis $p=0.004)$. Prandial boluses were given at the start of meal ingestion on both visits.

\section{Hypoglycemic events}

We saw no adverse events other than hypoglycemia. Eight participants (53\%) had at least 1 hypoglycemic event (plasma glucose concentration $<3.0 \mathrm{mmol} / \mathrm{L}$ ) during a control visit, whereas only 1 participant (7\%) had at least 1 hypoglycemic event during dual-hormone closed-loop delivery (McNemar test $p=0.02$ ), an 8-fold difference. For 7 participants (47\%), hypoglycemic events occurred only during the control visit; no participants had hypoglycemic events only during closed-loop delivery, 1 participant $(7 \%)$ had hypoglycemic events during both visits, and 7 participants $(47 \%)$ had no hyperglycemic events during either visit (data not shown). For the participant with hypoglycemia during both visits, 3 episodes occurred during the control visit and 2 episodes occurred during closed-loop delivery (data not shown). We saw a total of 12 hypoglycemic events during control visits and 2 events during closed-loop delivery (data not shown). We treated each event with $15 \mathrm{~g}$ oral carbohydrate. A second treatment was necessary for 4 events (all during control visits), 1 of which required a third treatment.

\section{Characteristics of glucagon delivery}

Total glucagon delivery during closed-loop delivery was 0.076 (IQR $0.016-0.170$ ) $\mathrm{mg}$ per visit (Appendix 3), resulting in a modest increase in mean plasma glucagon level $(66$ v. $56 \mathrm{pg} / \mathrm{mL}$, repeated measures analysis $p=0.01$; Table 1$)$. Glucagon was delivered intermittently (average of 1 minibolus every $3.6 \mathrm{~h}$ ) and in small amounts (0.014 [IQR 0.013-0.036] mg/bolus). Insulin delivery was suspended for $40( \pm 35)$ minutes before delivering glucagon but was not enough to prevent falling glucose levels (Figure 3). Glucagon delivery was used to prevent impending hypoglycemia rather than to treat it. The median plasma glucose level was $4.9 \mathrm{mmol} / \mathrm{L}$ and descending at a rate of $0.8 \mathrm{mmol} / \mathrm{L}$ per hour 
at the time of glucagon delivery, and $5.3 \mathrm{mmol} / \mathrm{L}$ and ascending at a rate of $1.5 \mathrm{mmol} / \mathrm{L}$ per hour 20 minutes after glucagon delivery (Figure 3 ). None of the participants reported any gastrointestinal symptoms after receiving glucagon boluses. Of the 61 glucagon miniboluses delivered, $24(40 \%)$ were given nocturnally. Insulin delivery before a glucagon minibolus was suspended for a longer time during the night compared with during the overall study period (57 min v. $40 \mathrm{~min}$ ). This was because, in case of impending hypoglycemia, glucose levels fell less rapidly during the night than during exercise or the late postprandial period.

\section{Sensor performance}

Sensor performance was adequate for closedloop delivery (relative absolute error $12.9 \%$ [IQR 6.5\%-21.6\%]). However, sensor readings often showed concentrations in the target range when plasma glucose was actually in the hyperglycemic range (sensor under-read by 1.07 [IQR $0.25-1.88] \mathrm{mmol} / \mathrm{L}$ in the hyperglycemic range [plasma glucose $>8.0 \mathrm{mmol} / \mathrm{L}$ ]; $n=555$ ). This may explain why the percentage of time spent above the target range was not reduced with closed-loop delivery. During closed-loop delivery, sensing errors possibly increased time spent above the target range by $10 \%-15 \%$ (the median percentage of time spent above the target range was $16 \%$ [data not shown] when calculated using sensor data, compared with $29 \%$ when calculated using plasma glucose measurements [Table 1]). We did not see sensor under- reading in the nonhyperglycemic range $(0.18$ [IQR -0.70 to 0.85 ] $\mathrm{mmol} / \mathrm{L}$ [plasma glucose $\leq$ $8.0 \mathrm{mmol} / \mathrm{L}] ; n=828)$. Improvements in sensor performance will likely further improve closedloop delivery.

\section{Interpretation}

Dual-hormone closed-loop delivery improved glucose control and reduced the risk of hypoglycemia in our 15 participants, as compared with continuous subcutaneous insulin infusion. Rates of hypoglycemia (plasma glucose concentration $<3.0 \mathrm{mmol} / \mathrm{L}$ ) were reduced, with no increased risk of hyperglycemia.

Hypoglycemia is common in type 1 diabetes, and its management is difficult because patients might not show symptoms. Hypoglycemia remains underestimated, with up to $60 \%$ of events going unrecognized. ${ }^{15}$ Recurrent hypoglycemia leading to hypoglycemia unawareness emphasizes the need for improved prevention and mitigation strategies. ${ }^{4}$ Hypoglycemia alarms are now an essential part of continuous glucose sensors, but they are of limited benefit during the night. ${ }^{16}$ Hypoglycemia is feared by most patients and remains the most common adverse effect of insulin therapy. Dual-hormone closed-loop delivery appears to have the potential to reduce the risk of hypoglycemia. Patients with hypoglycemia unawareness might benefit the most from this technology. However, this potential should be confirmed with larger and longer studies in an outpatient setting.

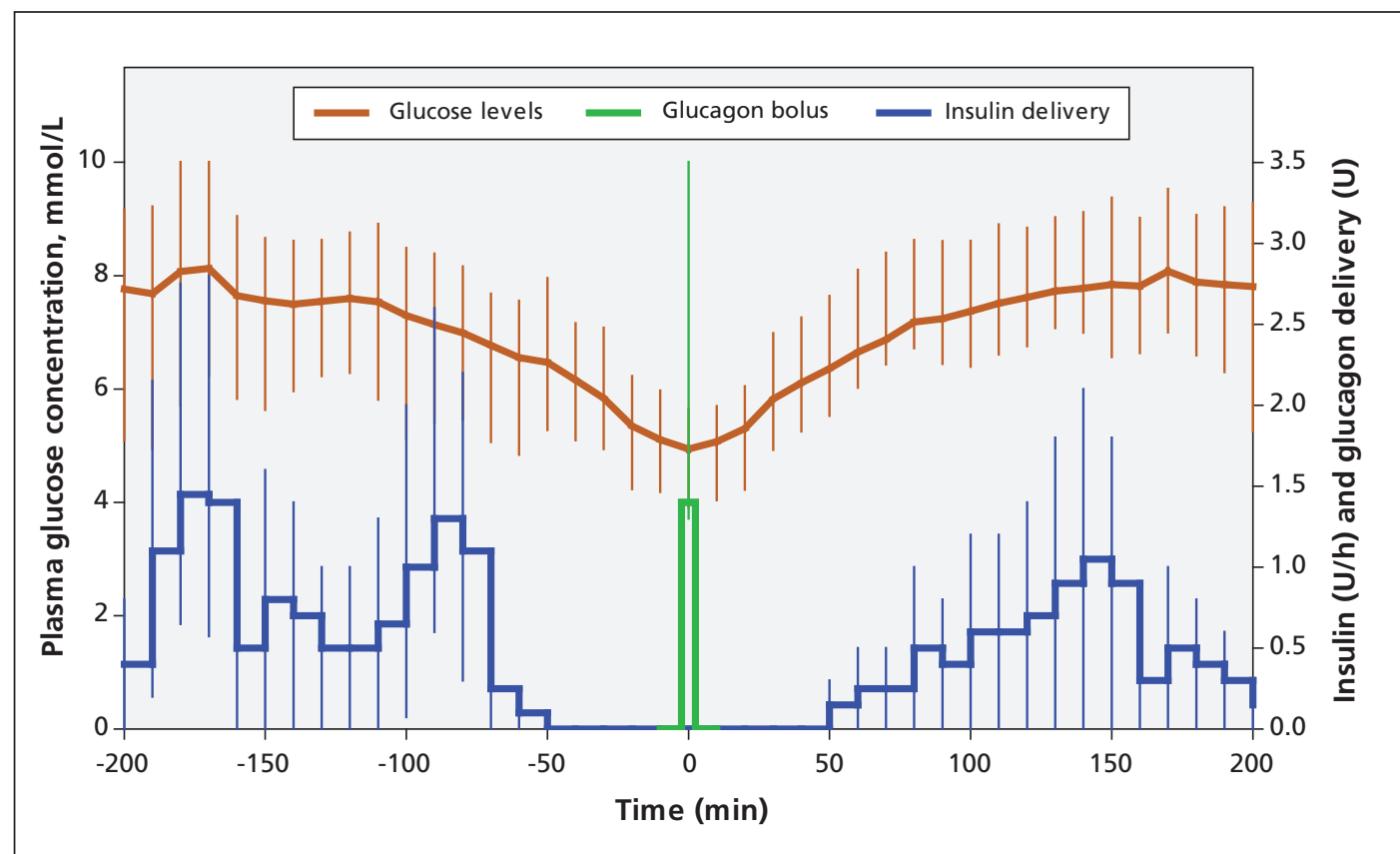

Figure 3: Profiles (medians and interquartile ranges) of plasma glucose concentration and basal insulin infusion before and after receipt of glucagon bolus ( $n=61$ boluses). 
Mean plasma glucose levels did not differ between the 2 treatments. However, betweenpatient variability in mean plasma glucose level was 50\% higher during control visits than during closed-loop delivery, suggesting higher dispersion of individual mean plasma glucose levels. Hypoglycemia was common during control visits and therefore reduced mean glucose levels, whereas closed-loop delivery eliminated most hypoglycemia without increasing mean glucose levels. This trend is also seen in the glucose profiles shown in Figure 1.

\section{Comparison with other studies}

Recent randomized trials involving adults with type 1 diabetes have shown that overnight closedloop insulin delivery increases the percentage of time for which plasma glucose levels are in the target range and decreases the time spent in the hypoglycemic range compared with conventional treatment. ${ }^{10,11}$ However, the reductions in time spent in the hypoglycemic range and numbers of patients with hypoglycemic events were not as remarkable as those in our study.

Although there have been previous studies of closed-loop insulin delivery ${ }^{17,18}$ and closed-loop insulin and glucagon delivery ${ }^{13,14,19}$ involving adults with type 1 diabetes, they did not have a randomized study design to make comparisons with conventional therapy. Furthermore, most of these studies have assessed the performance of the dosing algorithm rather than the clinical application of the whole system. Instead of a single glucose sensor, infusions were based on venous glucose ${ }^{14}$ or multiple sensors, ${ }^{13}$ and were sometimes overridden by an attending physician. ${ }^{13,17}$ Moreover, the glucose sensor was calibrated using referencequality venous plasma glucose $\mathrm{e}^{10,17,19}$ instead of capillary finger-stick measurements, potentially overestimating the benefits of closed-loop delivery. These issues may limit the application of the results of such studies in clinical practice. Our study avoided these shortcomings.

\section{Limitations}

Our study was powered using data from other studies of closed-loop delivery; ${ }^{11,13}$ despite having statistical significance in most outcomes, our study was limited by its small sample size. An additional limitation to our study was the absence of allocation blinding, but blinding participants to the interventions was practically challenging. Finally, current glucagon formulations are unlikely to be suitable for extended pump use, because they are unstable at room temperature after reconstitution. Research is underway to develop more stable formulations of the hormone..$^{20,21}$

\section{Conclusion}

Compared with conventional therapy, dualhormone closed-loop delivery improved shortterm glycemic control and reduced the risk of hypoglycemia among 15 adults with type 1 diabetes. Closed-loop delivery systems have the potential to substantially improve the management of diabetes and the safety of patients. These systems will probably be introduced gradually to clinical practice, with early generations focusing on overnight glucose control and using insulin alone. Their limitations and benefits will be elucidated, and their performance will be perfected over time.

\section{References}

1. The effect of intensive treatment of diabetes on the development and progression of long-term complications in insulin-dependent diabetes mellitus. The Diabetes Control and Complications Trial Research Group. N Engl J Med 1993;329:977-86.

2. Nathan DM, Cleary PA, Backlund JY, et al. Intensive diabetes treatment and cardiovascular disease in patients with type 1 diabetes. N Engl J Med 2005;353:2643-53.

3. Writing Team for the Diabetes Control and Complications Trial/Epidemiology of Diabetes Interventions and Complications Research Group. Sustained effect of intensive treatment of type 1 diabetes mellitus on development and progression of diabetic nephropathy: the Epidemiology of Diabetes Interventions and Complications (EDIC) study. JAMA 2003;290:2159-67.

4. Cryer PE. Hypoglycaemia: the limiting factor in the glycaemic management of type I and type II diabetes. Diabetologia 2002; 45:937-48.

5. Hypoglycemia in the Diabetes Control and Complications Trial; The Diabetes Control and Complications Trial Research Group. Diabetes 1997;46:271-86.

6. Choudhary P, Geddes J, Freeman JV, et al. Frequency of biochemical hypoglycaemia in adults with type 1 diabetes with and without impaired awareness of hypoglycaemia: no identifiable differences using continuous glucose monitoring. Diabet Med 2010;27:666-72.

7. Juvenile Diabetes Research Foundation Continuous Glucose Monitoring Study Group. Prolonged nocturnal hypoglycemia is common during 12 months of continuous glucose monitoring in children and adults with type 1 diabetes. Diabetes Care 2010;33:1004-8.

8. Bergenstal RM, Tamborlane WV, Ahmann A, et al. Effectiveness of sensor-augmented insulin-pump therapy in type 1 diabetes. $N$ Engl J Med 2010;363:311-20.

9. Hovorka R. Closed-loop insulin delivery: from bench to clinical practice. Nat Rev Endocrinol 2011;7:385-95.

10. Breton M, Farret A, Bruttomesso D, et al. Fully integrated artificial pancreas in type 1 diabetes: modular closed-loop glucose control maintains near normoglycemia. Diabetes 2012;61:2230-7.

11. Hovorka R, Kumareswaran K, Harris J, et al. Overnight closed loop insulin delivery (artificial pancreas) in adults with type 1 diabetes: crossover randomised controlled studies. BMJ 2011; 342:d1855.

12. Shichiri M, Kawamori R, Yamasaki Y, et al. Wearable artificial endocrine pancreas with needle-type glucose sensor. Lancet 1982;2:1129-31

13. Castle JR, Engle JM, El Youssef J, et al. Novel use of glucagon in a closed-loop system for prevention of hypoglycemia in type 1 diabetes. Diabetes Care 2010;33:1282-7.

14. El-Khatib FH, Russell SJ, Nathan DM, et al. A bihormonal closed-loop artificial pancreas for type 1 diabetes. Sci Transl Med. 2010;2:27ra27.

15. Chico A, Vidal-Rios P, Subira M, et al. The continuous glucose monitoring system is useful for detecting unrecognized hypoglycemias in patients with type 1 and type 2 diabetes but is not better than frequent capillary glucose measurements for improving metabolic control. Diabetes Care 2003;26:1153-7.

16. Buckingham B, Block J, Burdick J, et al. Response to nocturnal alarms using a real-time glucose sensor. Diabetes Technol Ther 2005; 7:440-7.

17. Kovatchev B, Cobelli C, Renard E, et al. Multinational study of subcutaneous model-predictive closed-loop control in type 1 diabetes mellitus: summary of the results. J Diabetes Sci Technol 2010;4:1374-81. 
18. Atlas E, Nimri R, Miller S, et al. MD-logic artificial pancreas system: a pilot study in adults with type 1 diabetes. Diabetes Care 2010;33:1072-6.

19. Russell SJ, El-Khatib FH, Nathan DM, et al. Blood glucose control in type 1 diabetes with a bihormonal bionic endocrine pancreas. Diabetes Care 2012;35:2148-55.

20. Steiner SS, Li M, Hauser R, et al. Stabilized glucagon formulation for bihormonal pump use. J Diabetes Sci Technol 2010;4:1332-7.

21. Ward WK, Massoud RG, Szybala CJ, et al. In vitro and in vivo evaluation of native glucagon and glucagon analog (MAR-D28) during aging: lack of cytotoxicity and preservation of hyperglycemic effect. J Diabetes Sci Technol 2010;4:1311-21.

Affiliations: From the Institut de Recherches Cliniques de Montréal (Haidar, Dallaire, Alkhateeb, Coriati, Messier, Rabasa-Lhoret); the Centre for Intelligent Machines, McGill University (Haidar, Boulet); the Montréal Children's Hospital (Legault, Millette); the Nutrition Department (Rabasa-Lhoret), Université de Montréal; and the Endocrinology Division (Rabasa-Lhoret), Montreal University Hospital, Montréal, Que.; and the Jaeb Center for Health Research (Cheng), Tampa, Fla.

Contributors: Ahmad Haidar and Rémi Rabasa-Lhoret coordinated the study. Ahmad Haider, Rémi Rabasa-Lhoret,
Laurent Legault, Benoit Boulet and Ammar Alkhateeb designed the study. Ahmad Haider, Maryse Dallaire, Rémi Rabasa-Lhoret, Laurent Legault, Adèle Coriati and Ammar Alkhateeb conducted the study. Ahmad Haidar designed and implemented the dosing algorithm. Ahmad Haider, Ammar Alkhateeb, Virginie Messier, Peiyao Cheng and Maude Millette analyzed the data. All of the authors contributed to the interpretation of the results, the writing and critical review of the manuscript, and the approval of final version submitted for publication.

Funding: This study was supported by Diabète Québec, the Canadian Diabetes Association (operating grant no. OG-111-3462-RR) and the J.-A. De Sève Chair held by Rémi Rabasa-Lhoret. Medtronic supplied the pumps and sensors used in the study, and read the manuscript before its submission. None of the sponsors had any role in the study design, data collection, data interpretation, or writing of the report.

Acknowledgements: The authors thank Annabelle Mathieu, Anne Bossy, Annie Tardif, Corinne Suppere, Sonia Fortin, Diane Mignault, Hortensia Mircescu and Agnès Räkel for their support in conducting the study.

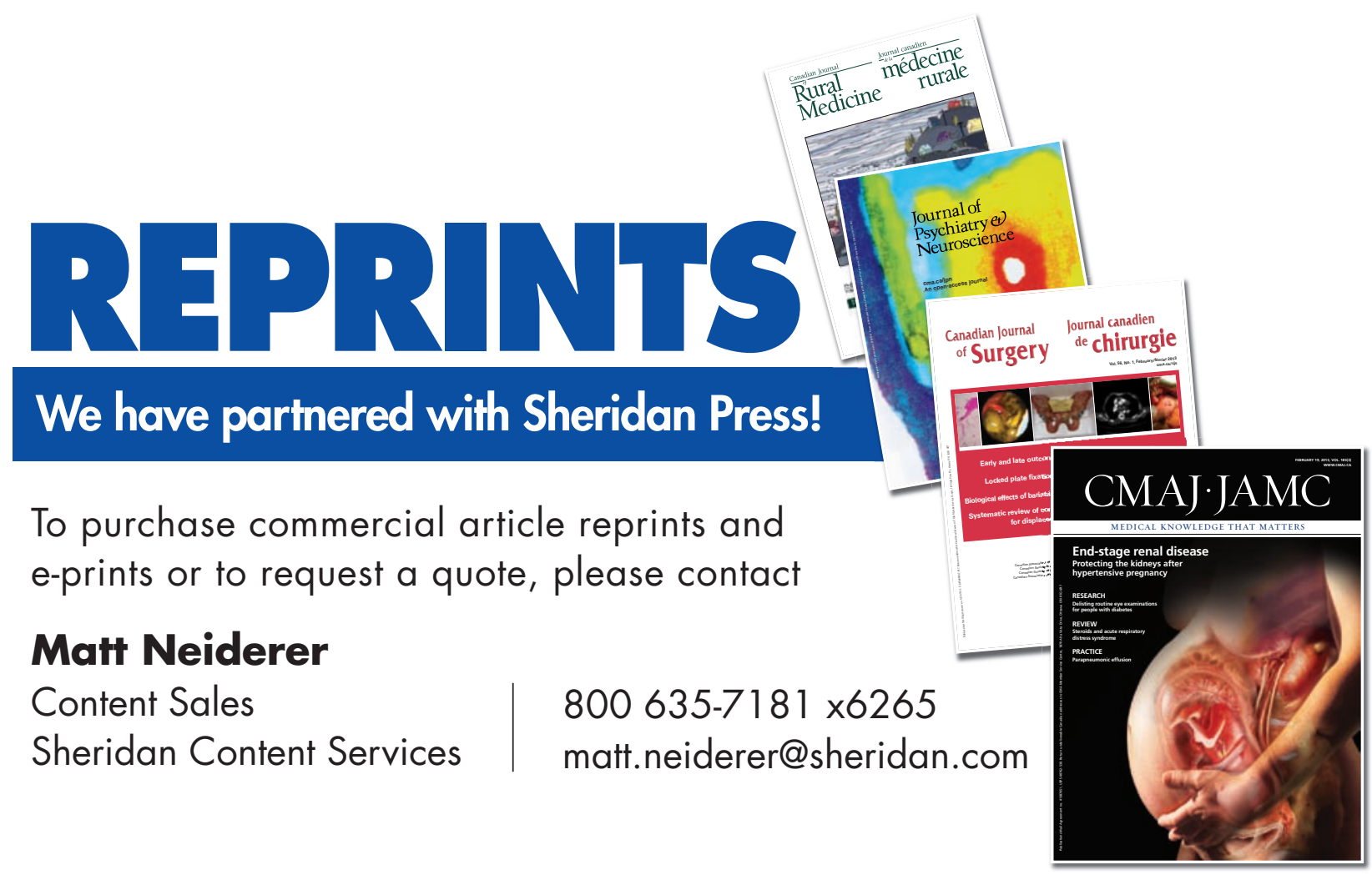

